Revista Educación 27(2): 67-77, 2003

\title{
TRABAJO SOCIAL Y EDUCACIÓN PRIMARIA. MEDIACIÓN HACIA UNA CULTURA DE PAZ
}

\author{
Norma Méndez Vega
}

\author{
Recibido 3-V-2003 • Aceptado 10-VI-2003
}

\begin{abstract}
Resumen: El presente artículo es un aporte para el trabajo social en la educación primaria. Destaca la importancia de trabajar a favor de una cultura de paz, y parte de que el ambiente de la educación primaria es propicio para este fin, ya que los niños y las niñas deben recibir la motivación hacia un mundo en el que la participación y el compromiso son elementos esenciales para promover la cultura de paz y calidad de vida. Ofrece algunos elementos teórico-metodológicos para que trabajo social se inserte en el ambiente y se constituya en mediador de procesos participativos con el resto de la comunidad escolar, constituída tanto por el personal docente, administrativo, padres, madres y equipos interdisciplinarios del Programa para el mejoramiento de la calidad de la educación y vida en las comunidades urbanas de atención prioritaria (PROMECUM).
\end{abstract}

Palabras clave: Política Educativa, Educación Primaria, Cultura de Paz, Trabajo Social, Equipo Interdisciplinario, Metodología Participativa.

\section{Introducción}

El Ministerio de Educación Pública de Costa Rica, a través del Programa para el mejoramiento de la calidad de la educación y vida en las comunidades urbanas de atención prioritaria (PROMECUM), ha implementado como uno de los componentes de este Programa la denominada Estrategia de Equipos Interdisciplinarios, en los cuales se encuentran laborando profesionales en trabajo social. Lo anterior, motiva el presente artículo, que pretende ofrecer un aporte al quehacer profesional de esta disciplina dentro del campo de la educación primaria, visualizando en ese quehacer la promoción de una cultura de paz. El trabajador social o la trabajadora social, son llamados a constituirse en facilitadores y mediadores en la promoción del diálogo y de acciones concretas, a partir de la incursión en los procesos administrativos de los centros educativos en donde laboran.

Esta reflexión es producto de la observación y el análisis, al compartir con grupos de trabajadores y trabajadoras sociales, que en la actualidad forman parte de estos equipos interdisciplinarios. Lo anterior gracias a la participación como investigadora en el proyecto denominado: El Trabajo Social en los Equipos de los Centros Educativos de Enseñanza Primaria. 
Los resultados de esta investigación, apuntan a la necesidad de apoyar las iniciativas de trabajo social para promover desde ahí, propuestas concretas de acciones tendientes a promover una cultura de paz.

Por tanto este artículo contiene un primer apartado que ubica la importancia de reflexionar sobre todo el trabajo que se hace en la educación primaria para el fomento de una cultura de paz. En la presentación de este tema se dan algunos principios que se consideran esenciales para este propósito, además de algunos cuestionamientos y aspectos sobre cultura de paz. En el segundo apartado, se habla de generalidades de la Política Educativa vigente y de PROMECUM. En el siguiente apartado se ubican algunos planteamientos acerca de PROMECUM y los espacios que ofrece dentro de la educación primaria para el ejercicio profesional del trabajo social. Posteriormente, se propone una estrategia metodológica como camino a seguir por trabajo social con los equipos interdisciplinarios. Por último algunos comentarios a manera de conclusiones.

\section{Educación primaria y cultura de paz}

La educación primaria se puede visualizar como un campo abierto y propicio para el fomento de la democracia y la cultura de paz. En el análisis del deber ser del sistema educativo, se pueden deducir algunos elementos esenciales, que en este documento se les denomina principios fundamentales y que tienen que ver con el trabajo arduo, pero necesario e interesante, de promover la cultura de paz; cultura que debe resurgir en la población infantil, como planta nueva y fuerte en sus raíces. Estos principios fundamentales son los siguientes:

- La democracia y la cultura de paz no son procesos que se aprenden de memoria y que ya están dados, sino que se construyen, en el entendido de que la paz, es un derecho y una responsabilidad.

- El sistema educativo requiere de un enfoque innovador y con una perspectiva de cambio constante, según los requerimientos de las personas que demandan sus servicios y que a la vez, son parte del mismo dentro de un contexto social específico.

- Se descarta completamente la discriminación, por condición de raza, por condición de género, por condición económica, por ubicación geográfica, entre otros.

- $\quad$ Debe permitir la plena participación de todas las personas en los diferentes momentos del quehacer educativo, de manera que permita el desarrollo individual y colectivo.

- El sistema educativo tiene, como una de sus tareas fundamentales, el ser formador de valores que trasciendan modas y diferencias sociales y económicas, y brinden una formación integral.

- $\quad$ El sistema educativo formal, por lo tanto, tiene un papel importante en la promoción de aquellos valores y normas que puedan hacer que el individuo crezca, pero no a costa de los demás sino con los demás; no en desigualdad de condición, sino en un sistema donde las oportunidades son iguales para todos.

- La plataforma o estructura escolar debe ser tal, que permita a los educandos formarse en libertad y democracia participativa. Que les permita "soltar" la imaginación y construir procesos de enseñanza aprendizaje 
más creativos, estimulantes, y satisfactorios para todos.

Es importante partir de la experiencia, del quehacer cotidiano, del sentir de los que participan en los procesos de educación y con todo este conocimiento, sugerir líneas de acción, sin imposiciones que "meten" a todas y todos en el mismo "cajón".

Es ir construyendo esa convicción profunda que crea y recrea los derechos y los deberes humanos. La niña y el niño pueden sentirse estimulados a compartir el aprendizaje, sus ideas, sus sueños con aquellas personas con las que comparte su vida escolar. Es un sistema educativo donde el diálogo es un requisito indispensable, acompañado de estrategias metodológicas que así lo promuevan y de un conocimiento y una actitud crítica ante la sociedad, que a pesar de que su sistema educativo plantea la justicia, la paz y la democracia, el entorno dice lo contrario. Es decir, viven un proceso de educación informal, en donde la violencia, la injusticia social, las desigualdades, la falta de respeto por la dignidad humana, son vivencias fuertes que muestran a todas luces y como normal, la inequidad.

De ahí que al analizar la institución escolar, es importante tener presente cuestionamientos tales como los siguientes:

- ¿El modelo pedagógico supera una dirección academicista y lineal con estrategias poco adecuadas en donde se le brinda poco tiempo al estudiante?

- ¿Tienen el grupo de los docentes y las docentes suficiente capacitación y tiempo para la reflexión y el análisis de su quehacer profesional?

- ¿La evaluación que se da en el centro educativo permite valorar la calidad de la educación que se imparte?
- ¿Cómo se operacionaliza el marco normativo y político sobre la regionalización educativa, para que facilite el ajuste de la educación a los requerimientos de cada región y propicie la participación comunal?

- $\quad$ ¿Se promueve la calidad educativa a través de: utilización de material didáctico, la informática, el incentivo a docentes y capacitación a los padres y madres de familia?

- ¿Es el director o directora del Centro Educativo un agente de cambio, facilitador y generador de acciones que logren ese fin?

- ¿Se ha mejorado la calidad de la enseñanza a través de la aplicación de nuevos métodos en el aula?

- ¿LLos aspectos burocráticos han obstaculizado la labor educativa?

- ¿Se ajusta la educación a los cambios sociales y a las diferencias socioculturales de la sociedad?

- $\quad$ ¿Se toman en cuenta los cambios y las necesidades de los niños y niñas, y se les educa para que sean sujetos activos con conocimientos, actitudes y destrezas para enfrenar su entorno social exitosamente?

- ¿Cuáles son los enfoques y las herramientas que utiliza la gestión institucional para facilitar un rendimiento óptimo de la organización?

Todas estas interrogantes, involucran a quienes laboran en un centro educativo, y el interés de este artículo es destacar el rol que tiene o puede tener el profesional y la profesional en trabajo social, que en este momento o en el futuro están o estarán incorporados a la educación primaria, como 
facilitadores y mediadores en la búsqueda de respuestas a las mismas.

De esta forma se pretenden compartir algunas inquietudes y pensamientos con respecto al quehacer del trabajo social como un mediador hacia una cultura de paz, la cual se fomenta a través de la participación plena. La educación tanto formal como no formal, es un medio que definitivamente contribuye a este proceso.

Fomentar una cultura de paz, es tarea que se inicia a partir de la enseñanza en derechos humanos, y esta enseñanza:

..."persigue educar a las personas para que aprendan a convivir en paz, respetando su dignidad personal y la de sus semejantes. Esta paz se genera con la armonía entre las personas y se relfeja en la tranquilidad del país. Es decir, un ciudadano educado como integrante de una familia, de una sociedad y de un país. Esto contribuye al buen funcionamiento y equilibrio de las instituciones que conforman la sociedad... Es así que consideramos que la educación para la paz es la mejor garantía para alcanzar la estabilidad democrática y la armonía que precisa y a la cual aspira la sociedad. De esta manera la escuela, es el espacio esencial donde las nuevas generaciones deben conocer, analizar, practicar y recibir tales valores democráticos con el propósito de construir una cultura de paz que emane del convencimiento profundo de cada persona. Estos conocimientos deben de llegar a realizarse de forma integral, es decir no sólo en la escuela sino también en la familia y en la sociedad. (Solís y Peñas, 1995, XIV y XV).

\section{Ubicación de trabajo social dentro de la política educativa hacia el siglo XXI}

El Estado costarricense "a partir de mayo de 1994 definió un cambio en la educación costarricense, sustentado en la legitimidad de un Sistema Nacional de Educación que sea orientador para el desarrollo social, político y económico del país.

Este renovado sistema educativo requiere de una visión de largo plazo en materia educativa y de un planteamiento de logros hacia la excelencia académica, respuesta en función de las demandas de la realidad nacional y de la nueva situación mundial a que se enfrentan los Estados Nacionales.
Esto exige un cambio en la manera de concebir la educación nacional como un proceso que trascienda las políticas de cada administración de gobierno, y demandar que para la educación se defina una política de Estado. Dentro de este contexto el Gobierno asumió una "política de sostenibilidad", a partir de la conferencia de Río de Janeiro, Brasil, en 1994." (Gómez, 2000:59).

Es así como en la actualidad (año 2003), aún se mantiene en vigencia, la denominada política educativa hacia el siglo XXI, que entre otros aspectos plantea: -una visión integral del ser humano, -coherencia entre teoría y práctica, -continuidad e innovación en las acciones educativas. Trata de generar los recursos humanos que elevarán la competitividad y productividad nacional para integrar exitosamente al país a la economía mundial. Es decir, plantea trascender la enseñanza centrada en el contenido, para que sea un instrumento eficaz que pueda crear oportunidades de participación y del posible cierre de la brecha entre los países y también entre las clases sociales, promoviendo la solución solidaria de los problemas.

Como fuente filosófica, esta política se centra en:

a. El Humanismo: El ser humano como razón de ser de la educación. Forma individuos con base en los principios de solidaridad y dignificación del ser humano.

b. El racionalismo: pretende que el individuo cultive su intelecto, sea creativo, participativo.

c. El constructivismo: cada individuo crea y recrea el conocimiento en su mente, y es gestor de su propio desarrollo.

Para hacer operativas estas intenciones, la política educativa se centra en seis aspectos fundamentales: 
1. "Planes y Programas.. serán una guía para los profesionales de la educación, padres de familia y estudiantes. Para los primeros deben constituirse en un norte para la evaluación, utilización de textos y recursos didácticos y en una respuesta para sus propias necesidades de formación, capacitación y educación continua....

2. La formación, capacitación y educación continua del Educador. El educador es una figura clave en la transformación de la educación costarricense, de ahí que la formación, capacitación y educación continua de éstos, pretende desarrollar la capacidad de los maestros y profesores para hacer frente a los retos que la política educativa plantea...

3. Evaluación. La evaluación cumple tres funciones bien delimitadas teóricamente: una diagnóstica, una formativa y una sumativa...

4. Los recursos didácticos... en sus múltiples modalidades (computadores, videos, materiales concretos, etc.) deben jugar un papel relevante en el proceso de construcción del pensamiento...

5. La mediación del Educador... desde el punto de vista de la estrategia didáctica, se define como la interacción entre el aprendiz y un experto...desarrollar habilidades y destrezas cognitivas y adquirir destrezas motoras, crear hábitos de reflexión y estimular con ello las actitudes hacia aprender a aprender, pensar y comprender....

6. Intermediación gerencial. Los actuales procesos administrativos, no corresponden a las condiciones en que se desenvuelve el país e implica en la mayoría de los casos, desmotivación para los clientes del sistema (docentes, padres de familia y educandos) al no recibir el trato que como personas y profesionales se merecen. Además la intermediación gerencial debe permitir que los escasos recursos se utilicen de la manera más racional posible." (MEP. EDU 2005. Resumen ejecutivo, 1996:9).

En fin pareciera que las condiciones se prestan para que la Educación sea una promotora de la cultura de paz, ya que plantea promover el desarrollo de individuos con una formación académica integral; trascender la enseñanza centrada en el contenido, para que sea un instrumento eficaz que pueda crear oportunidades de participación y de posible cierre de la brecha entre clases sociales, promoviendo la solución solidaria de los problemas.

Por otra parte, desde el punto de vista económico, la educación se encargaría de generar los recursos humanos necesarios para el desarrollo del país, dentro de un contexto de avance y apertura tecnológica. Además, se propone fortalecer valores y actitudes hacia el desarrollo social, en un marco de respeto por la diversidad cultural, social y étnica y un sentido de respeto hacia la preservación del ambiente. Lleva así a la participación y compromiso de directores, educadores, educandos, padres y madres de familia, en los destinos de la nación. Así mismo, se lograría que las decisiones políticas no estén solamente en manos de un grupo, sino que, la sociedad civil tenga más acceso a la toma de decisiones por medio de la participación.

A la par de esta política educativa y en correspondencia con sus postulados, se desarrolla también, en el año 1994 el Programa para el mejoramiento de la calidad de la educación y vida en las comunidades urbanas de atención prioritaria (PROMECUM). Es un programa que surge en la Administración Figueres Olsen dirigido específicamente para la atención de las comunidades con mayores necesidades del país. Su misión es:

"fortalecer y facilitar una educación de calidad, con una visión holística y un enfoque del desarrollo integral del ser humano. Desde el punto de vista filosófico propicia un ser humano solidario, crítico y autónomo, su desarrollo curricular se nutre de un enfoque constructivista, racionalista y humanista a la vez, que permite educar y formar integralmente a los niños y las niñas, de tal modo que los capacite para forjar su propio desarrollo, en lo económico, en lo social y en lo cultural." (Espinoza y Chévez:1999:2).

Este programa tiene tres componentes básicos: a) docentes Tutores, quienes se encargan de la promoción y capacitación permanente de las docentes y los docentes; b) Proyecto de Recuperación Integral (PRI), 
que se encarga de dar apoyo a estudiantes con dificultades en su proceso de aprendizaje; c) La estrategia de los equipos interdisciplinarios, que es la instalación en cada centro educativo donde se desarrolla PROMECUM, de un equipo de profesionales constituido por las disciplinas de psicología, orientación y trabajo social, sociología o promoción social. El equipo está conformado por tres personas donde se da el campo exclusivo a psicología y orientación, mientras que el tercer campo puede ser trabajo social, sociología o promoción social. Sin embargo hasta la fecha, existen más profesionales en trabajo social que en las otras dos disciplinas.

Como se puede apreciar PROMECUM es coherente con la política educativa vigente y es un programa que le abre un espacio a trabajo social. Surge entonces la pregunta: ¿qué pueden hacer las profesionales y los profesionales trabajo social para aprovechar este espacio en la educación primaria? A continuación se plantea una propuesta.

\section{Formas en que el trabajo social puede aprovechar su espacio en la educación primaria}

El trabajo social como disciplina y ejercicio profesional, tiene un amplio espacio para su ejercicio en el área social; precisamente la riqueza de la profesión es la gama de espacios para su accionar.

El Trabajo Social como:

“...una disciplina de las ciencias sociales que profesionalmente se ocupa de intervenir en problemas sociales que son el resultado de necesidades no satisfechas o contingentes, para crear conjuntamente con los sectores afectados, las condiciones para mejorar su calidad de vida y modificar las condiciones que generan estos problemas" (Catálogo Escuela de Trabajo Social, 2000:6),

mantiene una dirección del ejercicio profesional, cual es la participación con los grupos sociales en desventaja social y en diferentes y variados espacios como por ejemplo: salud, educación, seguridad social, justicia, entre otros.

De esta forma el trabajo social en la educación primaria apoya o debe apoyar las diferentes iniciativas para impulsar mejores condiciones de vida, sobre todo en aquellos niños, niñas y sus familias integrantes de sectores vulnerables y pobres de la sociedad.

PROMECUM le da un espacio a trabajo social, y hay que aprovecharlo como punto de partida para la acción profesional. Por lo tanto ante la pregunta qué hace o qué puede hacer este profesional, no se trata de dar un listado de acciones puntuales, sino sugerir una serie de pautas de acción que permitan abrir espacios para incursionar en la totalidad del ambiente escolar como una forma de contribuir en la promoción de la cultura de paz de la cual se habla en este artículo.

Si se retoman los principios fundamentales mencionados al inicio y se constituyen en la base epistemológica para la acción, el programa de trabajo debe contener las siguientes intenciones:

- Incursionar en el análisis de las formas de realizar los procesos de Planificación, Control, Supervisión y Evaluación del centro educativo donde está laborando, con el fin de buscar conjuntamente, nuevos planteamientos que lleven a la innovación y el cambio constante en administración educativa, con la participación de docentes, estudiantes, padres y madres.

- $\quad$ Conocer, mediante acercamientos incesantes, los sentimientos, valoraciones y esperanzas que las autoridades de la institución tienen sobre su práctica administrativa, para analizar con ellos o ellas, las posibilidades de una educación participativa y transformadora.

- Promover la elaboración conjunta de propuestas estratégicas para cubrir 
las áreas de acción que les asigna PROMECUM a los equipos interdisciplinarios, a saber: estudios diagnósticos e investigación, asesoría y capacitación a docentes, prevención integral, atención a situaciones psicosociales específicas. Todo lo anterior, de acuerdo al contexto en el cual se desenvuelven.

- Analizar la participación que tienen docentes, estudiantes, padres y madres de familia, en la gestión administrativa, con miras a fortalecerla.

Con base en estas intenciones, se hace también importante que se valore para sí y para los demás, algunos elementos que están presentes en la vida de las personas como lo son: las convicciones personales, la creatividad, el tipo de comunicación que se da, el desarrollo de habilidades y destrezas, el análisis de la realidad, el interés por la formación, autoformación y transformación de los educandos.

Como se aprecia, es necesario un trabajador social o trabajadora social que integre en su organización una perspectiva amplia y comprensiva de diferentes personas y elementos que interactúan, para generar una acción planificada y coherente con las necesidades de la población meta de cada centro educativo. El Gráfico 1 resume estos aspectos.

Trabajo social es así una disciplina facilitadora para la promoción de un proceso dinámico dentro de la institución, con miras a la existencia permanente de una actitud de innovación. Actúa en unión con los otros actores y actoras, para procurar un ambiente escolar que propicie la consolidación de una cultura de paz, en medio de la sociedad en la que se desenvuelve.

Lo anterior implica una clara intención de acercarse a las diferentes personas actoras en el centro educativo donde labora y asumir un rol de mediación para fortalecer el diálogo y la motivación hacia acciones concretas que involucran a toda la comunidad educativa, a partir, tanto de las fortalezas que poseen, como las necesidades y problemas que enfrentan.

Esta práctica de la mediación, permite al profesional en trabajo social acercarse a los otros actores y actoras del proceso educativo y partir de los conocimientos de

Gráfico 1

Aspectos a contemplar en la planificación de trabajo social en el campo de la educación primaria

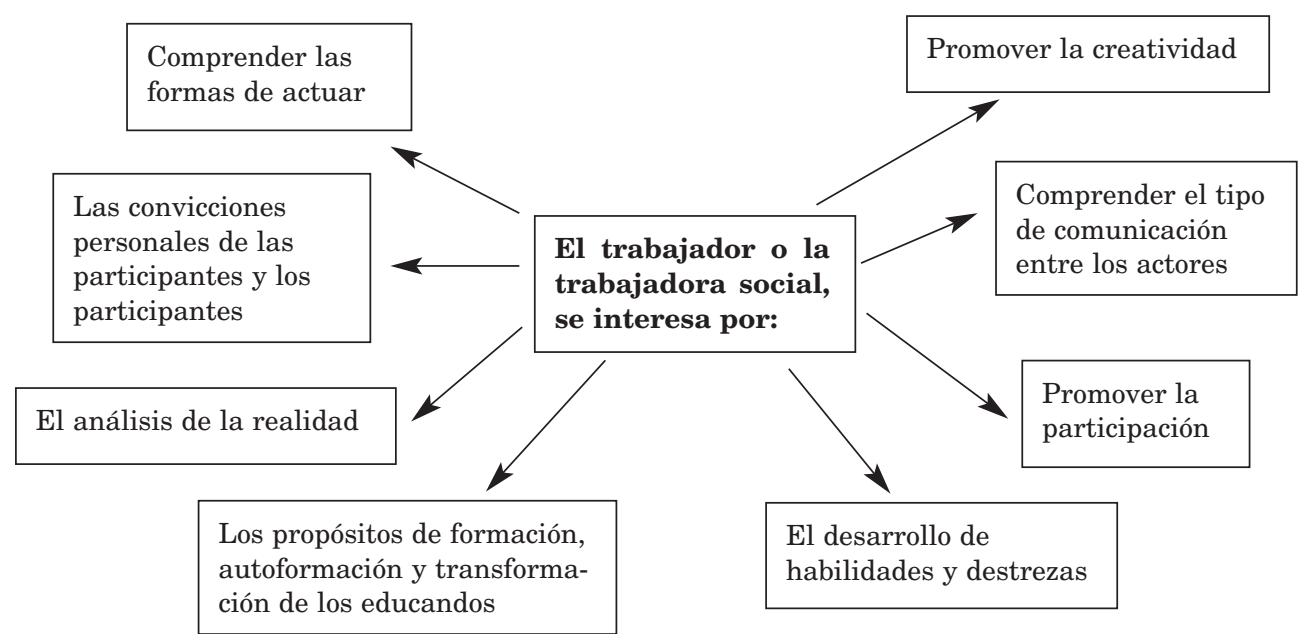

Fuente: Elaboración propia. 
todas las personas involucradas, lo mismo que a partir de la forma en que transmiten ese conocimiento y de las acciones que proponen con miras a una transformación.

Esta es la estrategia metodológica con una perspectiva participativa, desde donde abarca todo el conjunto de personas y procesos, donde la operacionalización de los mismos a través de diferentes acciones y actores, se dan de acuerdo al contexto específico desde el cual se quieran implementar, es decir, desde cada centro educativo.

En síntesis se puede decir que la trabajadora social o el trabajador social dentro del campo de la educación primaria tiene las siguientes herramientas para su acción profesional:

1. A partir de las áreas de intervención asignadas por PROMECUM: estudios diagnósticos e investigación, asesoría y capacitación a docentes, prevención integral y atención de situaciones psicosociales específicas.

2. Tiene como fundamento epistemológico cinco principios fundamentales:

a) La construcción de procesos de democracia y cultura de paz, b) Un enfoque de educación innovadora de acuerdo a las demandas de contexto social específico, c) Ningún tipo de discriminación, d) Plena participación de las personas involucradas, e) Toma en cuenta que la escuela es formadora de valores.

3. Se constituye en un facilitador o facilitadora y mediador o mediadora del proceso administrativo del centro educativo al cual pertenece.

4. Implementa una estrategia metodológica participativa en donde el saber, la práctica y las propuestas de cambio de todas y todos los involucrados, son un punto de partida esencial.

\section{A modo de propuesta ${ }^{1}$}

Como un aporte a esta estrategia metodológica, se propone un posible camino a seguir, pero en el entendido, tal y como se dijo anteriormente que su operacionalización depende del contexto específico.

Se pueden definir dos etapas fundamentales: 1) Consolidación de los equipos interdisciplinarios. 2) Acercamientos a la realidad y a la población involucrada en la vida cotidiana del centro educativo.

\section{Consolidación de los equipos interdisciplinarios}

El trabajador social o la trabajadora social, tiene claro que no trabaja de forma aislada y por lo tanto debe tomar en cuenta a las otras personas del equipo. $\mathrm{Su}$ rol protagónico es en el sentido de liderar este proceso, partiendo de un papel importante en la facilitación y mediación de las acciones.

Cada equipo interdisciplinario debe hacer una revisión profunda de elementos como los siguientes: su origen, las políticas a las que están respondiendo, las acciones que han desarrollado y están desarrollando hasta el momento, sus logros, necesidades, fortalezas teóricas, metodológicas y como punto importante, una revisión de cuánto y qué conocen del contexto en el cual laboran, tanto a nivel intra escolar como comunal. Es importante que revisen su proceso como equipo, cuánto se conocen, qué hace cada persona miembro del equipo, sus sentimientos y valoraciones como personas y como profesionales desde su propia disciplina. De esta reflexión surgen las propuestas para fortalecerse como equipo.

En el campo teórico es importante que el equipo conozca sobre: Teoría de la educación, derechos humanos y cultura de paz, administración educativa. En el campo metodológico: los diferentes métodos participativos. 


\section{Acercamientos a la realidad y a la población involucrada en la vida cotidiana del centro educativo}

Esta etapa se puede dividir en tres fases:

Primera Fase: Acercamiento inicial a la comunidad escolar

Elaborar un plan de acercamientos sucesivos a docentes, directores, estudiantes, padres, madres, con el propósito de conocer los sentimientos, valoraciones y esperanzas de estas personas con respecto a la vida en la escuela. Lo anterior con el objetivo de obtener una visión más profunda sobre quienes son, qué esperan, qué proponen y cuáles son sus fortalezas para apoyar un cambio dirigido a fortalecer la cultura de paz, a partir del trabajo con la población infantil.

De esta primera aproximación surgen una serie de espectativas que trabajo social y el resto del equipo interdisciplinario puede traducir en temas para implementar la segunda fase.

\section{Segunda Fase: Tematización}

Esta fase implica retomar el producto de la fase anterior y traducirlo en ejes temáticos y la conformación de unidades pedagógicas para ser presentados y analizados por la población meta: docentes, autoridades del centro educativo, estudiantes, padres, madres de familia.

Es una fase de trabajo arduo, a partir de un conocimiento claro de las necesidades y espectativas de las diferentes personas actoras del proceso educativo. El equipo interdisciplinario se aboca a preparar esos ejes temáticos para, posteriormente, en una tercera fase desarrollarlos en círculos de estudio o de análisis.

Supone el conocimiento del contexto, el estudio de las temáticas, la elaboración de material didáctico para los diferentes grupos.

Es una fase donde los equipos interdisciplinarios tienen la oportunidad de prepararse para llegar a estos grupos con temas de interés para los mismos, ya que han surgido de una consulta previa. Por tanto, desde esta óptica, se puede trabajar con la intención de modificar o cambiar actitudes, acciones, valoraciones, ya que este diálogo de saberes, va a permitir que se ponga en el tapete de la discusión aquello que las personas saben al desplegar sus acciones en torno a los procesos educativos; agregan un elemento importante cual es: cómo lo hacen, es decir sus estrategias para poner en práctica ese saber y, luego como último aspecto, aportan elementos que lleven a propuestas de cambio o transformación en el trabajo por una cultura de paz.

Con estos aportes el equipo interdisciplinario elabora material, llámese módulos, reflexiones, o cualquier otro nombre, al conjunto de temas con sus respectivas estrategias metodológicas participativas, para acercarse en la fase siguiente al análisis conjunto.

Tercera fase: Análisis y programación para la acción

Esta fase implica programar momentos de reunión con los diferentes grupos (docentes, autoridades escolares, estudiantes, padres y madres) para realizar círculos de estudios y analizar las temáticas preparadas por el equipo interdisciplinario. Esta fase permite un análisis crítico y la implementación de propuestas para los cambios deseados. Surgen propuestas de acción conjuntas que se constituyen en los insumos para la programación y acción de los equipos interdisciplinarios con la participación de toda la población involucrada. Esta programación implica un riguroso y constante trabajo de planificación, ejecución, control y evaluación. Además de contar con una serie de técnicas e instrumentos para fomentar 
la participación. Requiere también de la voluntad política para realizar un trabajo que visto de esta perspectiva, puede contribuir a legitimar a los equipos interdisciplinarios en las escuelas, ya que se valora un trabajo conjunto donde cada persona sabe por qué y para qué se hace; además propicia que se tome en cuenta el contexto a partir de las necesidades, pero también de las fortalezas que poseen. Por otra parte tiene una orientación clara e importante hacia el fomento de la cultura de paz, que es el tema de fondo de esta propuesta. Además trabajo social y el resto del equipo interdisciplinario, tienen así la oportunidad de realizar diagnósticos, investigar, asesorar y capacitar.

\section{Reflexiones finales}

Vale la pena el esfuerzo por trabajar con miras a promover la cultura de paz, y vale todavía más la pena, trabajar esta perspectiva desde los centros educativos de primaria. Son los niños y las niñas quienes mueven y dan sentido a las instituciones educativas. Es alrededor de ellos y ellas donde convergen docentes, personas expertas en administración educativa, padres y madres de familia, que desean un mejor futuro para sus hijos e hijas y otro personal como en este caso, los equipos interdisciplinarios.

Es de vital importancia el esfuerzo que hagan trabajo social, sociología, psicología y orientación, para cumplir con la misión que les fue encomendada de fortalecer y facilitar una educación de calidad, con una visión holística y un enfoque del desarrollo integral del ser humano. Y más aún, si todo ese esfuerzo fuera sólo por fomentar la cultura de paz, estarían contribuyendo para que todas las personas que concurren en el centro educativo miren la vida con ojos de solidaridad, criticidad y autonomía, bases sólidas para una sociedad justa, democrática y por lo tanto en paz.
Los procesos participativos que trabajo social impulse y facilite, le permiten dar sentido a su espacio profesional en este campo de la educación primaria y en consecuencia, son una base que les da legitimidad.

El sistema educativo como campo para la disciplina de trabajo social, le abre el espacio para que trabaje por la paz, vista ésta como un derecho y una responsabilidad. Además le da un contexto específico, que es la circunstancia y el lugar donde labora, y le demanda que descarte todo tipo de discriminación, por condición de raza, por condición de género, por condición económica, por ubicación geográfica, entre otros, permitiendo así la propiciación del desarrollo individual y colectivo y la formación de valores que trasciendan modas y diferencias sociales y económicas, y brinden una formación integral.

Con esta oportunidad que ofrece el sistema educativo el trabajador social o la trabajadora social que ha tenido el privilegio de insertarse en un centro educativo de primaria y trabajar en equipo, debe sentir satisfacción por la misión que ha venido cumpliendo y por la gama inmensa de posibilidades que tiene para ver el trabajo futuro con la esperanza de contribuir a una sociedad mejor.

\section{Notas}

1. Esta propuesta es una adaptación de la propuesta que realiza Boris Yopo P., especialista en Educación y Desarrollo y consultor del Centro Regional de Educación de Adultos y Alfabetización Funcional para América Latina (CREFAL).

\section{Referencias bibliográficas}

Chéves, Alberto; Espinoza Eugenio. "Programa para el mejoramiento de la calidad de la educación y vida en 
las comunidades urbanas de atención prioritaria”. Ministerio de Educación Pública, San José, Costa Rica. 1999.

Gómez F., Mónica. La conflictividad Institucional en Procesos de Reforma Educativa y sus implicaciones sociales en Escuelas Públicas de la Regional Educativa Cartago, Costa Rica. Tesis de posgrado en Trabajo Social. Universidad de Costa Rica. 2000 .
Ministerio de Educación Pública. "Política Educativa hacia el siglo XXI. Resumen ejecutivo". 1996.

Solís, Luis Guillermo y Peñas, Mercedes. Educación para la PAZ. Editorial EUNED, San José, Costa Rica. 1995.

Universidad de Costa Rica. Catálogo Escuela de Trabajo Social. 2000.

Yopo, Boris. Metodología de la Investigación participativa. CREFAL. México. 1985.

Norma Méndez Vega

Profesora de la Escuela de Trabajo Social, Universidad de Costa Rica 